\title{
A Computational Modelling for the Ag Nanoparticles Coalescence Process: A Case of Surface Plasmon Resonance
}

Giovani M. Faccin, Miguel A. San-Miguel, Juan Andrés, Elson Longo, and Edison Zacarias da Silva J. Phys. Chem. C, Just Accepted Manuscript • DOI: 10.1021/acs.jpcc.7b00769 • Publication Date (Web): 08 Mar 2017

Downloaded from http://pubs.acs.org on March 9, 2017

\section{Just Accepted}

"Just Accepted" manuscripts have been peer-reviewed and accepted for publication. They are posted online prior to technical editing, formatting for publication and author proofing. The American Chemical Society provides "Just Accepted" as a free service to the research community to expedite the dissemination of scientific material as soon as possible after acceptance. "Just Accepted" manuscripts appear in full in PDF format accompanied by an HTML abstract. "Just Accepted" manuscripts have been fully peer reviewed, but should not be considered the official version of record. They are accessible to all readers and citable by the Digital Object Identifier (DOI®). "Just Accepted" is an optional service offered to authors. Therefore, the "Just Accepted" Web site may not include all articles that will be published in the journal. After a manuscript is technically edited and formatted, it will be removed from the "Just Accepted" Web site and published as an ASAP article. Note that technical editing may introduce minor changes to the manuscript text and/or graphics which could affect content, and all legal disclaimers and ethical guidelines that apply to the journal pertain. ACS cannot be held responsible for errors or consequences arising from the use of information contained in these "Just Accepted" manuscripts. 


\title{
A Computational Modelling for the $\mathrm{Ag}$
}

\section{Nanoparticles Coalescence Process: A Case of Surface Plasmon Resonance}

\author{
Giovani M. Faccin, ${ }^{* \dagger}$ Miguel A. San-Miguel, ${ }^{\ddagger}$ J. Andres, ${ }^{\complement}$ E. Longo, ${ }^{\S}$ and E. Z. da \\ Silva ${ }^{*,}, \|$ \\ Universidade Federal da Grande Dourados, Universidade Estadual de Campinas, \\ Universitat Jaume I, and Universidade Federal de São Carlos \\ E-mail: GiovaniFaccin@ufgd.edu.br; zacarias@ifi.unicamp.br
}

\footnotetext{
*To whom correspondence should be addressed

†Faculdade de Ciências Exatas e Tecnológicas, Universidade Federal da Grande Dourados - Unidade II, CP 533, 79804-970, Dourados - MS, Brazil.

†Instituto de Química, UNICAMP, CP 6165, 13083-970, Campinas - SP, Brazil.

`Departament de Química Física i Analítica, UJI-Universitat Jaume I, Av. de Vicent Sos Baynat, s/n, Castelló de la Plana 12071, Spain.

${ }^{\S}$ CDMF-UFSCar-Universidade Federal de São Carlos, P.O. Box 676, CEP, 13565-905 São Carlos-SP, Brazil.

"Instituto de Física "Gleb Wataghin", UNICAMP, CP 6165, 13083-9859, Campinas - SP, Brazil. 


\begin{abstract}
Motivated by recent transmission electron microscopy (TEM) experiments on $\alpha-\mathrm{A}_{2} \mathrm{WO}_{4}$, the coalescence process of Ag nanoparticles (NPs) is investigated using molecular dynamics (MD) simulations. These $\mathrm{Ag}$ NPs are formed by irradiation of $\alpha-\mathrm{Ag}_{2} \mathrm{WO}_{4}$ crystals by electrons from a TEM gun. This behavior can be considered as a clear example of surface plasmon resonance (SPR), in which Ag NP coalescence processes are controlled by dipole-dipole interaction forming larger clusters. The interactions between Ag NPs along the coalescence processes are studied using MD simulations with embedded atom method (EAM) effective potentials for Ag. With these choices of methods coalescence is studied addressing different scenarios for the interacting NPs, all possible to occur in experiments.
\end{abstract}

\title{
Keywords
}

plasmons, nanoparticles, coalescence, silver, molecular dynamics, computer simulations

\section{Introduction}

In the past 20 years, imaging and manipulation of structures, at atomic level, are mainly fueled by the fast developments of new experimental techniques and computational methods. Irradiation with an electron beam is known to be an effective technique for changing or modifying materials in the high vacuum system of the electron microscope. Electron miscroscopies provide a robust technique to observe static and dynamic structures at atomic and nanoscale level, and also provoke both structural and chemical rearrangements. ${ }^{1}$

Recent advances in transmission electron microscopy (TEM) technology enables studies of atomic scale processes involving from single atoms to nanoparticles and nanowires, yielding novel nanostructures, ${ }^{2}$ especially for those that cannot be fabricated using conventional chemical and physical methods. Therefore, these new applications that TEM has made can 
be considered as a versatile tool for materials engineering at the nanoscale. The above processes were triggered by electron beam irradiation which allowed simultaneous fabrication and observation in a convenient and, more importantly, direct manner. Jiang ${ }^{3}$ summarizes a variety of beam damage phenomena relating to oxides in (scanning) transmission electron microscopes, and underlines the shortcomings of currently popular mechanisms. In particular, very recently, Mannan et al. ${ }^{4}$ reported that electron-beam irradiation has been used to fabricate nanoparticles and nanorods on Ag surfaces with nanometric precision under exposure to the electron beam. Both high control over nanorod dimensions and high placement accuracy have been demonstrated. This observation is a clear manifestation of an electronsolid interaction and motivated by the recent discovery of the formation of Ag nanoparticles (NPs) and nanowires due to the electron beam irradiation by a TEM gun on $\alpha-\mathrm{A} g_{2} \mathrm{WO}_{4}$ crystals, ${ }^{5,6}$ we investigate the nature of the formation mechanism under the guidance of computer simulations. Reasons for the formation of these new structures have been given in recent publications. ${ }^{7,8}$

Sintering and welding of metal nanorods and NPs have been investigated recently, both experimentally ${ }^{9-11}$ and also computationally. ${ }^{12}$ For example, Grouchko et. al. ${ }^{13}$ have studied welding and coalescence processes of $\mathrm{Ag}$ nanostructures on $\mathrm{Au}$ and $\mathrm{Au}$ on $\mathrm{Ag}$ NPs experimentally and with the aid of computer simulations. Other studies considered the important question of oriented attachment in $\mathrm{Au} \mathrm{NPs}^{14}$ and coalescence process in Pd NPs. ${ }^{15}$ The present work interprets many scenarios of the interaction of Ag NPs emerging when $\alpha-\mathrm{A}_{2} \mathrm{WO}_{4}$ crystals are irradiated by an electron beam ${ }^{6,16,17}$

Both Ag and Au NPs, with diameters of less than $100 \mathrm{~nm}$ can be excited into surface plasmon resonance (SPR) states due to a laser field. This is a very well known effect with a wide range of applications dating from the IV century, the famous Lycurgus Cup being a very striking example, ${ }^{18}$ in which $\mathrm{Ag}$ and $\mathrm{Au}$ NPs around $70 \mathrm{~nm}$ in diameter dispersed in the glass reflect green light and transmit red light. TEM experiments have elucidated this interesting effect. These metal NPs are also responsible for the beautiful coloring of many 
medieval windows in famous European churches. Au NPs, due to this plasmonic effect, have also been used in a very promising application of cancer therapy. ${ }^{19}$

Electron beam spectroscopies have advantages in spatial resolution and accessibility to areas of interest. In particular, the electron gun used for TEM imaging of nano-objects such as NPs was demonstrated in TEM and in electron energy loss spectroscopy (EELS) measurements to produce plasmonic effects similar to laser irradiation. ${ }^{20-23}$ The electron beam carries an associated electric field that, for the correct frequency, similarly to the case of laser light, excites SPR in the NPs. SPRs that appear in metal NPs are associated with collective oscillations of free electrons and occur in the visible part of the electromagnetic spectrum. A particular NP becomes a neutral dipole due to the SPR. The interaction of nearby NPs occurs due to the neutral dipole-dipole interaction since this behavior is very similar to bonding and anti-bonding dimer nodes in molecules. A dipole pair can be in the dipole active mode, called bright mode, which corresponds to attractive interaction as well as in the dark mode, which corresponds to a repulsive interaction.

Systematic experimental and theoretical studies of the production of Ag nanorods and NPs upon electron irradiation of $\alpha-\mathrm{Ag}_{2} \mathrm{WO}_{4}$ crystals under the TEM experiments have opened a new avenue of interesting possibilities for the production of Ag NPs. ${ }^{5-8}$ The present article continues the discussion of the formation of new Ag nanostructures presented in those experiments with special emphasis on new effects of Ag NPs-NPs interactions caused by the same process of electron gun irradiation of the $\alpha-\mathrm{Ag}_{2} \mathrm{WO}_{4}$ crystals during imaging. In particular, experiments in refs ${ }^{16}$ show images of Ag NPs and their interactions forming other larger Ag NPs. Faccin et al. ${ }^{17}$ argued that SPR understood from Mie theory and neutral dipole interactions could be used to interpret and explain the observed effect. They also used molecular dynamics (MD) simulations to discuss the experimental images.

The simulations analysis presented in Faccin et. al. ${ }^{17}$ focused on understanding the experiments with interesting conclusions. The experiments produced many types of Ag NPs, ranging from almost perfect Wulff shaped Ag NPs, spherical crystalline Ag NPs, as well as 


\section{Computational Details}

We studied the interactions of Ag NPs that are attracted to each other in coalescence events. The focus is a setup where Ag NPs are produced in very diverse forms, with sizes from 4 to $10 \mathrm{~nm}$ in diameter, ranging from crystalline ordered spherical fcc Ag NPs, and also partially ordered (ordered Ag NPs with disordered regions and many stacking faults) spherical Ag NPs. After producing these different types of Ag NPs we studied their interaction and coalescence processes.

The present study, based on the experimental observations of our research group, is focused on the coalescence process due to the SPR neutral dipole formation. Ag NPs under the presence of the TEM electron beam feel the associated electric field that induces the formation of SPRs. The Ag NPs become nano-dipoles and when pairs of these Ag NPs are oriented in the so called bright mode, it corresponds to a case of attractive dipole-dipole interactions. Ag NPs approach each other and start the contact to proceed with the coalescence processes. In order to investigate the events that take place once the Ag NPs are produced in these experiments, this work uses MD simulations performed using the LAMMPS package ${ }^{24-27}$ and the embedded atom model $(\mathrm{EAM})^{28-31}$ with the parameterization for Ag from Sheng et . al. ${ }^{32}$ These two choices, namely LAMMPS and EAM are appropriate since we are studying 
large NPs and MD in the LAMMPS version, with an EAM effective potential which gives a description that is the closest to DFT one can obtain for systems of this size and time scale. In particular the EAM choice ${ }^{32}$ for the $\mathrm{Ag}$ effective potential is quite accurate, well documented and has been used extensively before. ${ }^{33-35}$

In the first simulation set, called crystalline ones, Ag NPs of spherical shape were cut from the bulk structure and then relaxed at 0K; after relaxing, the structures were approached to provide the initial point for sintering simulations; the dimer structures were then heated to the reported temperatures. In the second set, called annealed ones, the previous set of nanoparticles were heated to $1400 \mathrm{~K}$, which is over their fusion point, and then annealed to $300 \mathrm{~K}$ along 1 ns resulting in structures with less order and stacking faults which are more similar to real Ag NPs from the experiments we are attempting to discuss. After this process the samples are ready to be the starting point for their respective sintering simulations. For each set of simulations canonical ensemble MD simulations were used with Nosé-Hoover thermostat chains. ${ }^{36-38}$ Visualizations were created using the VMD and Ovito packages ${ }^{39-41}$ and the common neighbor analysis (CNA) of Dana et al. ${ }^{42}$ The equations of motion were integrated using a time step of $1 \mathrm{fs}$, which guaranteed negligible numerical deviations for the total duration of the simulations, whose typical duration was 20 ns. This was long enough to obtain equilibrated systems and to observe the processes that we discuss in the next section.

\section{Results and Discussion}

The studies reported in the present work involved the analysis of coalescence process of $\mathrm{Ag}$ NPs of different sizes in the range of 4 to $10 \mathrm{~nm}$. Therefore, we considered Ag NPs of 4.5 $\mathrm{nm}$ and $9.0 \mathrm{~nm}$. In order to understand different aspects of these interactions we studied Ag NPs of equal size, namely 4.5-4.5 nm and 9.0-9.0 nm. To study the effect of different sizes we also studied interactions of Ag NPs with sizes of 4.5 and $9.0 \mathrm{~nm}$. We performed simulations of completely ordered Ag NPs as well as partially disordered Ag NPs. These simulations 
were performed at 500K. We also studied the final stages of these Ag NP-Ag NP coalescence processes as function of temperature.

\section{Crystalline ordered NPs interaction}

We considered two sizes of equally sized crystalline Ag NPs, namely $4.5 \mathrm{~nm}$ and $9.0 \mathrm{~nm}$ NPs. Figure 1 shows the evolution of two $4.5 \mathrm{~nm} \mathrm{Ag} \mathrm{NPs} \mathrm{along} \mathrm{the} \mathrm{coalescence} \mathrm{process.} \mathrm{The} \mathrm{top}$ panel shows two sequences of images, the top images display the atomic form evolving, while the bottom sequence shows a planar cut of the same snapshots using CNA analysis. First images (a) (top and bottom) show the two Ag NPs before contact; the second image (b) displays the images after starting the coalescence process and shows a disordered interface produced by the contact of the Ag NPs; it also shows some stacking faults. The fact that the Ag NPs were ordered before colliding is important since this helps healing the structure back to the ordered structure. It is important to note that the stacking faults that were also generated have large energy barriers and are difficult to transform back to fcc, in fact the small stacking faults evolve, propagating all throughout the interface merging the two previous Ag NPs. The disordered regions get well ordered in the process either transforming into longer stacking faults or back into fcc. To make these statements more quantitative we calculated the radial distribution function $g(r)$ corresponding to the images from the top panel, the $g(r)$ corresponding to the snapshots are displayed at the bottom panel. These curves show a first well ordered $g(r)$, characteristic of a crystalline Ag NPs, (a). Upon touching (b) a typical disordered function emerges. As the evolution unfolds (c and d) some order is restored with the final $g(r)$ (e) almost as ordered as the starting structures, as can be seen from the CNA structure (e) with some remaining stacking faults.

Figure 2 shows the coalescence process of Ag NPs with sizes of $9.0 \mathrm{~nm}$. In this case, due to the larger particle size the disordered region that is formed during the collision is small compared with the Ag NP with size of $4.5 \mathrm{~nm}$, together with a small stacking fault that evolves back into fcc and a larger staking fault. A comparison with previous results on Ag 


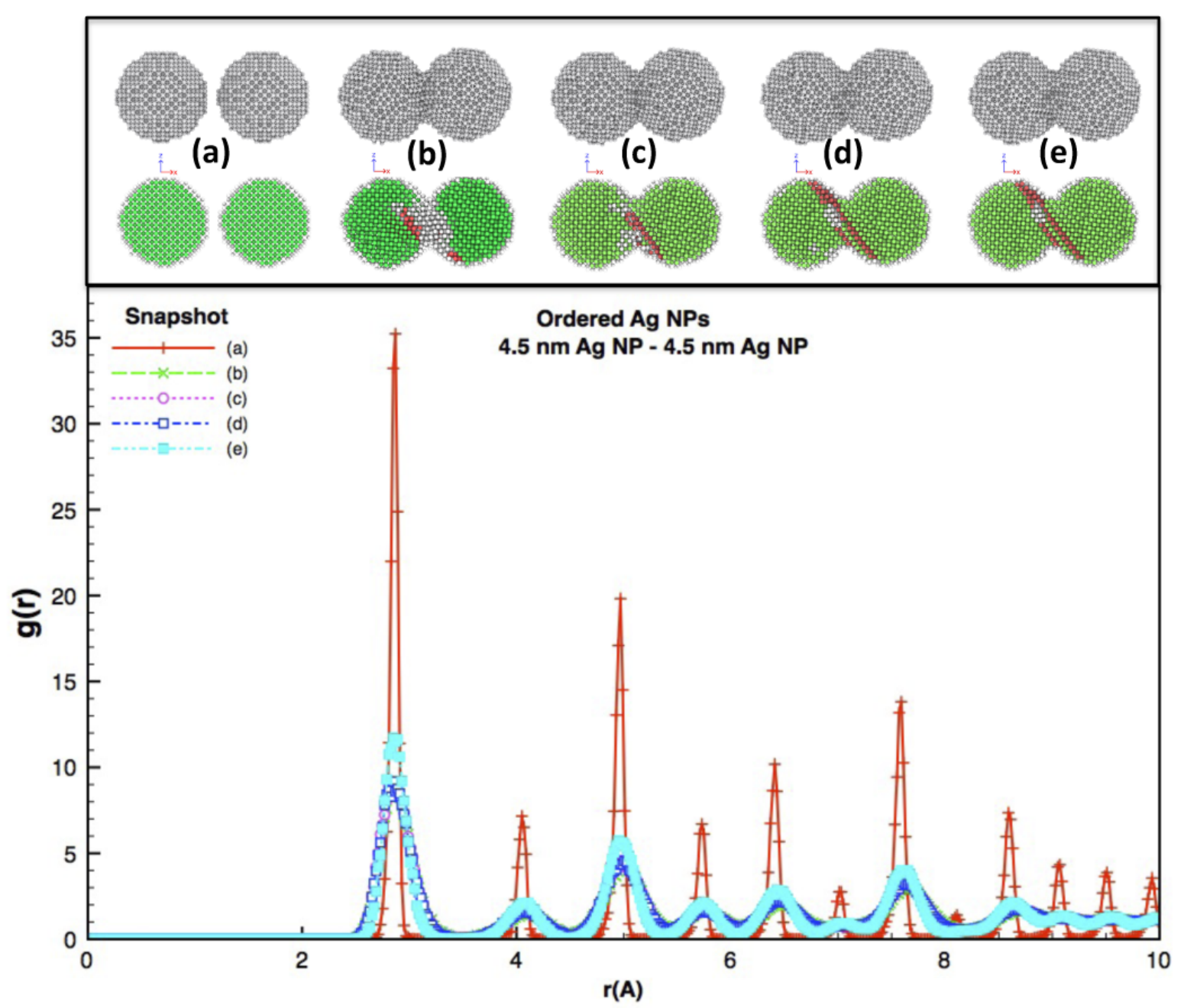

Figure 1: Simulation of the coalescence process of two $4.5 \mathrm{~nm}$ ordered Ag NPs at $500 \mathrm{~K}$. Top panel shows snapshots (a to e) of the structural evolution of the coalescence (upper sequence) and the CNA plots (lower sequence) that depict how the structure evolves. From ordered fcc NPs (green), during coalescence they form a disordered contact region (grey) and some hcp stacking faults (red) that evolve reordering and propagating stacking faults. Bottom panel shows the radial distribution function $g(r)$. 
Figure 2: Simulation of the coalescence process of two ordered $9.0 \mathrm{~nm} \mathrm{Ag} \mathrm{NPs} \mathrm{at} 500 \mathrm{~K}$. (a to d) (top line): structural evolution of the coalescence and (bottom line) the CNA plots that depict how the structure evolves from ordered fcc NPs (green). During coalescence they form a small disordered contact region (grey) and some hcp stacking faults (red) that evolve reordering and propagating small stacking faults.

NPs of $4.5 \mathrm{~nm}$ NPs renders the appearance of a well ordered new structure with a smaller stacking fault region. The $g(r)$ (SM-2 shown in the Supplementary Material) shows that after the first disorder formation (b) stacking fault formation and recovery of order occurs but are all small effects. Ordered structures tend to restore order in the coalescence process. This fact can be further inferred from the result of the different size NPs coalescence process. Figure 3 presents the coalescence process of a $4.5 \mathrm{~nm} \mathrm{Ag} \mathrm{NP}$ with a $9.0 \mathrm{~nm} \mathrm{Ag} \mathrm{NP.} \mathrm{In} \mathrm{this}$ case the fact that particles have different sizes causes an interesting event where as in the previous cases a disordered interface region develops upon contact (b) with stacking faults mostly in the small NP. Since order tends to drive order, the large ordered Ag NP drives order into the smaller one with the result that the new particle gets well ordered in the process. The $g(r)$ (SM-3 displayed in the SM) shows the emergence of order within the final structure.

The coalescence processes in ordered Ag NPs are very interesting but we do not think that these types of processes take place in the experiments. The experimentally produced $\mathrm{Ag}$ NPs due to electron beam irradiation by a TEM gun on $\alpha-\mathrm{A} g_{2} \mathrm{WO}_{4}$ crystals produces 


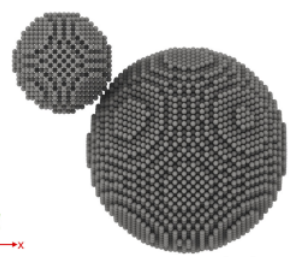

(a)

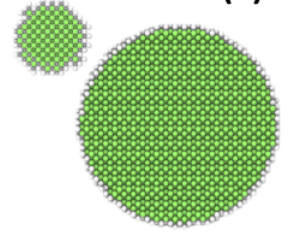

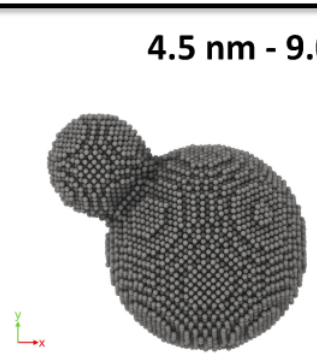

(b)

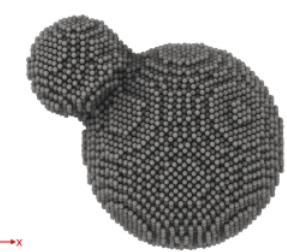

(c)

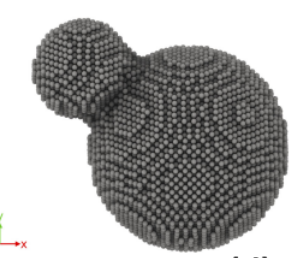

(d)
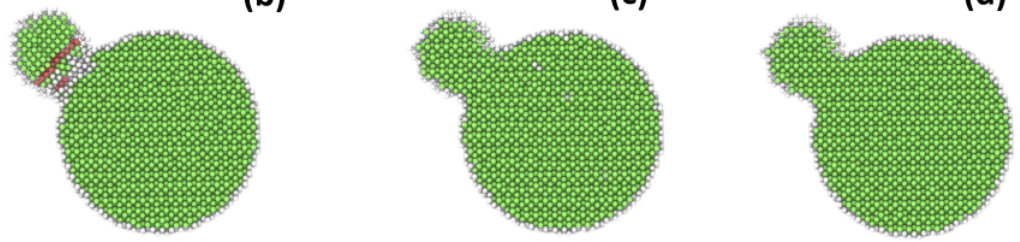

Figure 3: Simulation of the coalescence process of an ordered $4.5 \mathrm{~nm} \mathrm{Ag} \mathrm{NP}$ and a $9.0 \mathrm{~nm}$ Ag NP at $500 \mathrm{~K}$. Frames a to d display (top line) structural evolution of the coalescence and (bottom line) the CNA plots that depict how the structure evolves from ordered fcc NPs (green), during coalescence they form a small disordered contact region (grey) which develops and some hcp stacking faults (red) that evolve reordering into a perfectly ordered new particle.

more disordered structures.

\section{Disordered Ag NPs interaction}

Ag NPs can be produced with different degrees of order and diverse shapes ranging from spherical to well shaped Wulff constructions. The Ag NPs produced during the $\alpha-\mathrm{Ag}_{2} \mathrm{WO}_{4}$ experiments are not ordered, instead they show ordered regions as well as disordered and stacking fault regions. In order to learn how partially ordered Ag NPs evolve in coalescence processes we produced partially ordered Ag NPs by heating the ordered Ag NPs over their melting temperature and then annealed them back to $300 \mathrm{~K}$. These results are used for the next computer experiments, where we repeat the same studies performed for the ordered Ag NPs.

Figure 4 displays the coalescence of two partially ordered $4.5 \mathrm{~nm} \mathrm{Ag} \mathrm{NPs.} \mathrm{The} \mathrm{top} \mathrm{panel}$ shows the evolution of the structure similarly to the case of the ordered structures discussed previously. The first left images (a) show the two Ag NPs before contacting, top images 


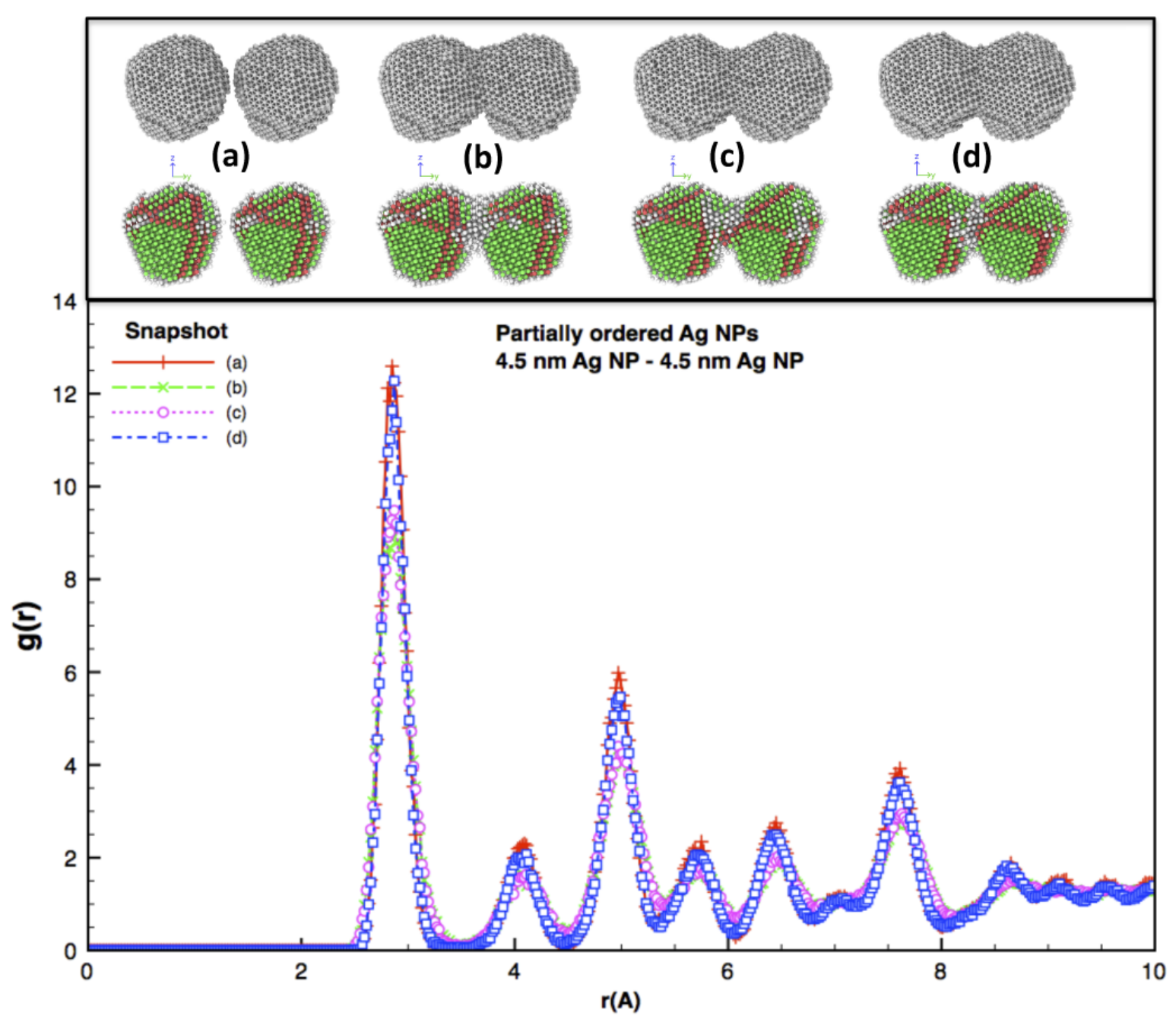

Figure 4: Simulation of the coalescence process of two partially ordered $4.5 \mathrm{~nm} \mathrm{Ag} \mathrm{NPs} \mathrm{at}$ $500 \mathrm{~K}$. Top panel shows snapshots (a to d) of the structural evolution of the coalescence (upper sequence) and the CNA plots (lower sequence) that depict how the structure evolves. During coalescence they form a disordered contact region (grey) and some hcp stacking faults (red). The new Ag NP evolves propagating stacking faults and reordering. Bottom panel shows the radial distribution function $g(r)$ of this evolution. 
shows the NP's structure and bottom their CNA cut. It can be seen that the Ag NPs start with ordered regions, disordered regions as well as many stacking faults. Upon contact they produce a disordered interface with propagation of stacking faults at the interface. The competition of disordered interface with surrounding stacking faults prevents healing to be as effective as in the case of ordered Ag NPs. The new structure that started with partially ordered structures again evolves to restore some of the order. This can be clearly seen in the $g(r)$ displayed at the bottom panel. In fact, as the size of the Ag NP increases the energy barrier created by the stacking fault becomes so large that it will block the healing process, leading to a disordered final stage.

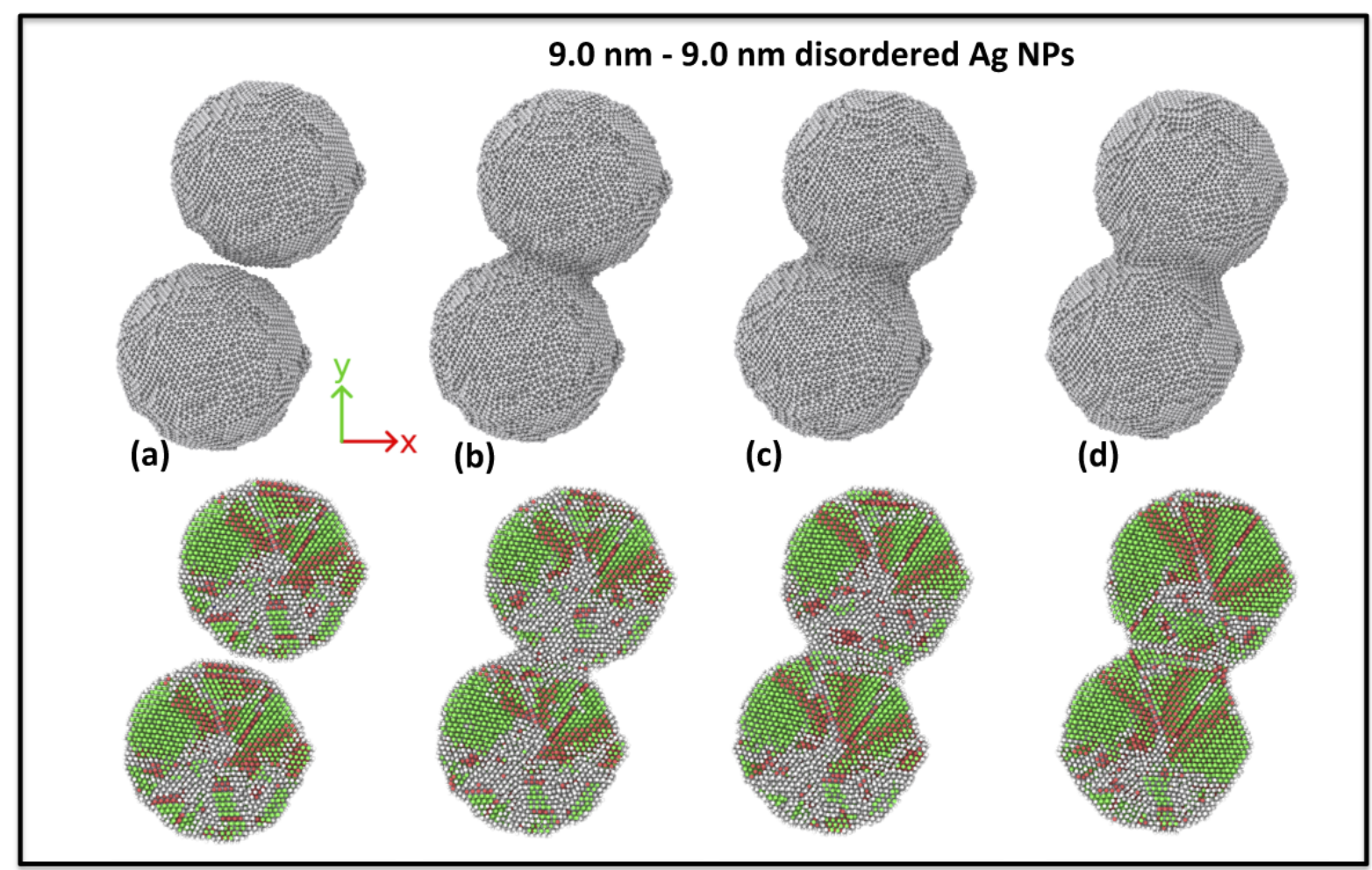

Figure 5: Simulation of the coalescence process of two partially ordered $9.0 \mathrm{~nm} \mathrm{Ag} \mathrm{NPs}$ at $500 \mathrm{~K}$. Frames a to d display (top line) the structural evolution of the coalescence and (bottom line) the CNA plots that depict how the structure evolves; during coalescence they form an amorphous contact region (grey) and some hcp stacking faults (red) that evolve reordering and healing some stacking faults.

The coalescence process of the larger Ag NPs, with 9.0-9.0 nm of size, is depicted in 
Figure 5. The initial partially ordered Ag NPs have large disordered regions and large stacking fault regions. Upon contacting a large disordered interface develops. For these NPs sizes the ordered fcc planes and the stacking fault regions force ordering reducing the disordered interface more efficiently, with a final Ag NP much more ordered with crystalline fcc and hcp regions. This recovery of order can clearly be seen in the $g(r)$ shown in the SM (SM-4). The case of different Ag NPs sizes in Fig. 6 ( 4.5-9.0 nm Ag NPs) shows a

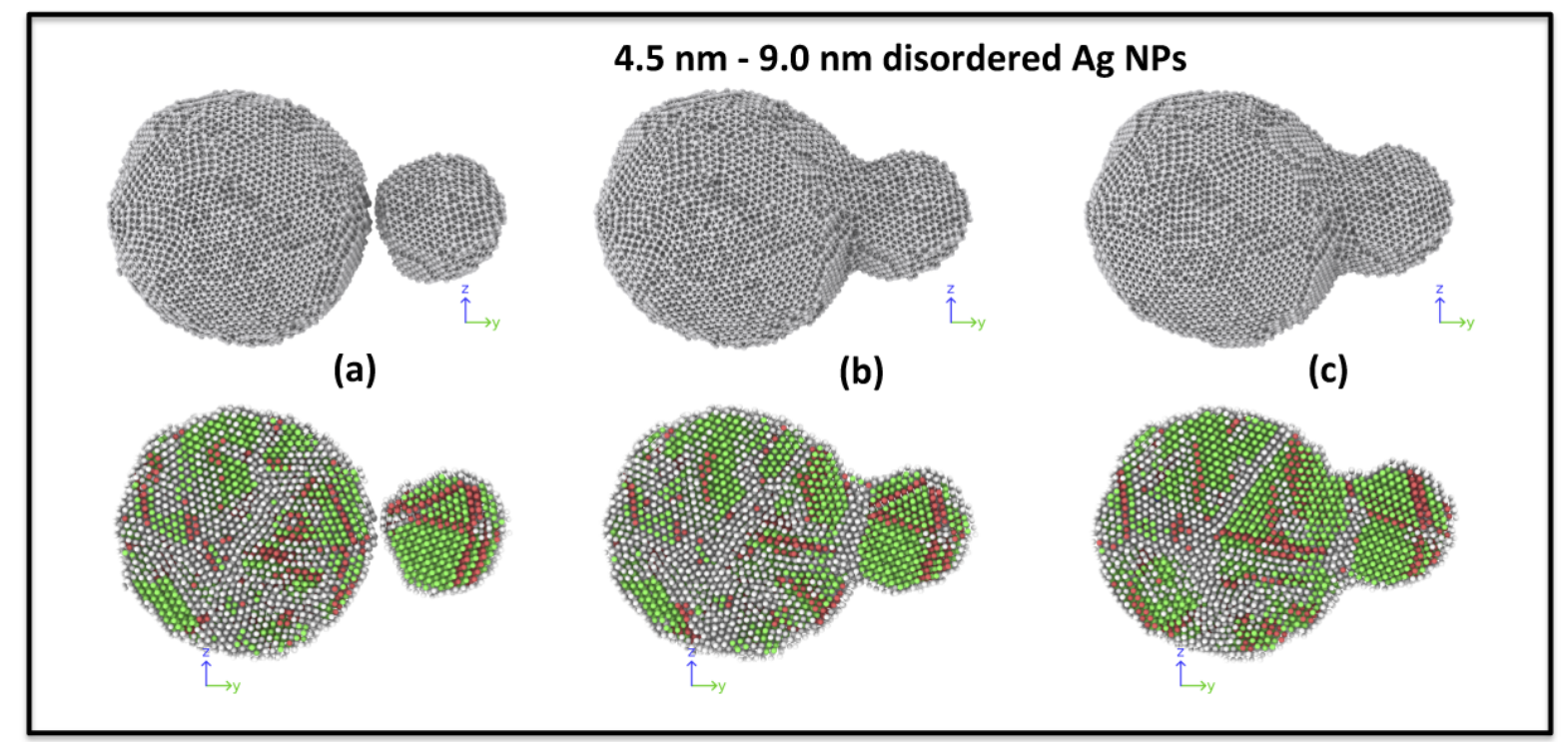

Figure 6: Simulation of the coalescence process of two partially ordered Ag NPs of $4.5 \mathrm{~nm}$ and $9.0 \mathrm{~nm}$ at $500 \mathrm{~K}$. Frames a to c display (top line) structural evolution of the coalescence and (bottom line) the CNA plots that depict how the structure evolves, during coalescence they form an amorphous contact region (grey) and some hcp stacking faults (red) that evolve reordering and healing some stacking faults.

disordered interface which in this case is not driven back to the ordered phases; the sintering process promotes some ordering in both sides of the new combined Ag NP with reduction of disordered regions. In the case of disordered Ag NPs the ordering is not as effective as it was in the case of ordered Ag NPs discussed in Figure 3. Nevertheless, some order is restored in the whole structure as well as ordering induced in what was the small Ag NP, i.e. there is a restructuring of some fcc region with the suppression of some stacking faults. This healing into order can be seen from the $g(r)$ shown in the SM (SM-5). One strong 
trend in all these cases is the evolution of stacking faults and the recovery of order. This particular simulation shows trends similar to the experimental sequence presented in the SM (see SM-1) as a motivation for the present work.

\section{Temperature effects}

The previous discussion was for a fixed temperature similar to the experiments. The effect of temperature in the sintering processes is also something worth investigating; therefore we discuss here also thermal effects. The coalescence process of crystalline Ag NPs is presented in Figures 7, 8 and 9. In Fig. 7 we present the evolution in temperature of the final stages

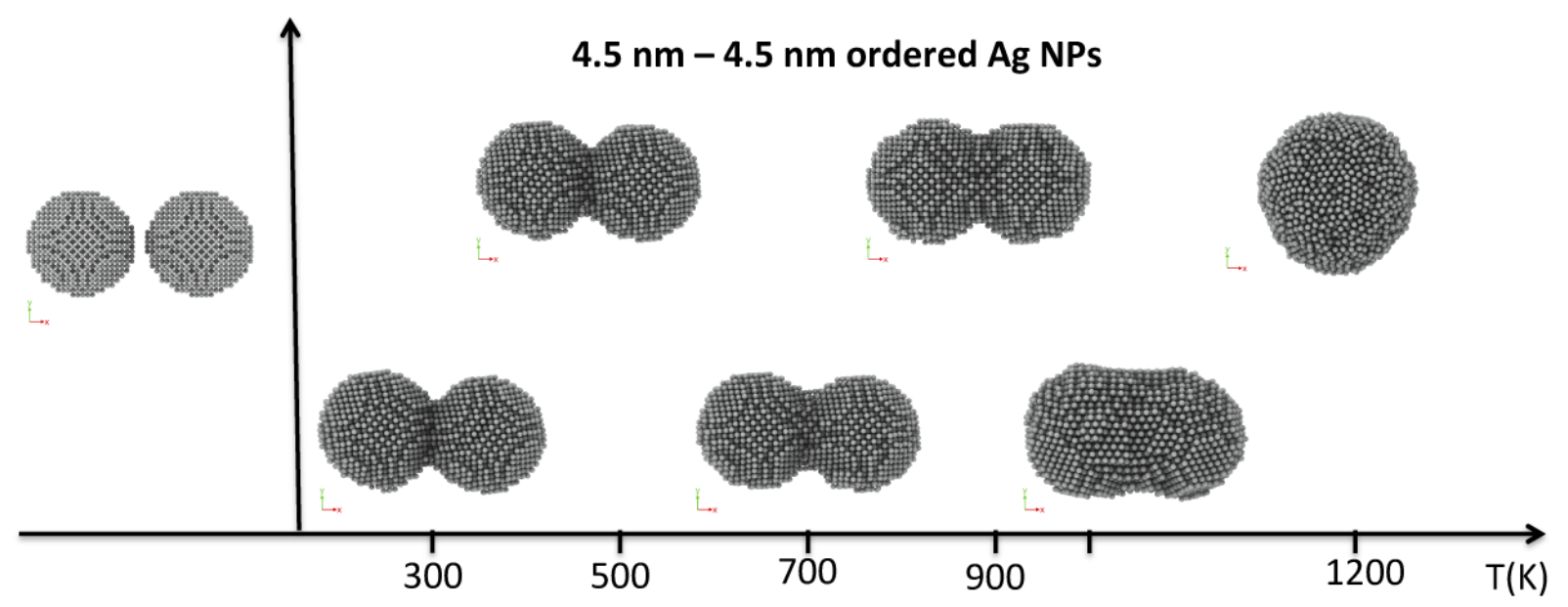

Figure 7: Temperature dependence of the coalescence process of two ordered Ag NPs of 4.5 nm.

of coalescence runs for temperatures from $300 \mathrm{~K}$ to $1200 \mathrm{~K}$ for $\mathrm{Ag}$ NPs of $4.5 \mathrm{~nm}$ of size. The effect of temperature drives the coalescence process to form well-rounded Ag NPs for high temperatures and is very effective at this size range. Fig. 8 shows the same sequence for equal size $9.0 \mathrm{~nm} \mathrm{Ag} \mathrm{NPs.} \mathrm{In} \mathrm{this} \mathrm{case} \mathrm{the} \mathrm{temperature} \mathrm{helps} \mathrm{to} \mathrm{evolve} \mathrm{the} \mathrm{coalescence}$ process but is less effective than the previous case. Fig. 9 presents the 4.5-9.0 nm Ag NP's coalescence. The temperature effect helps sintering where the smaller Ag NP tends to be incorporated into the larger one. The temperature effect in the case of disordered NP's 


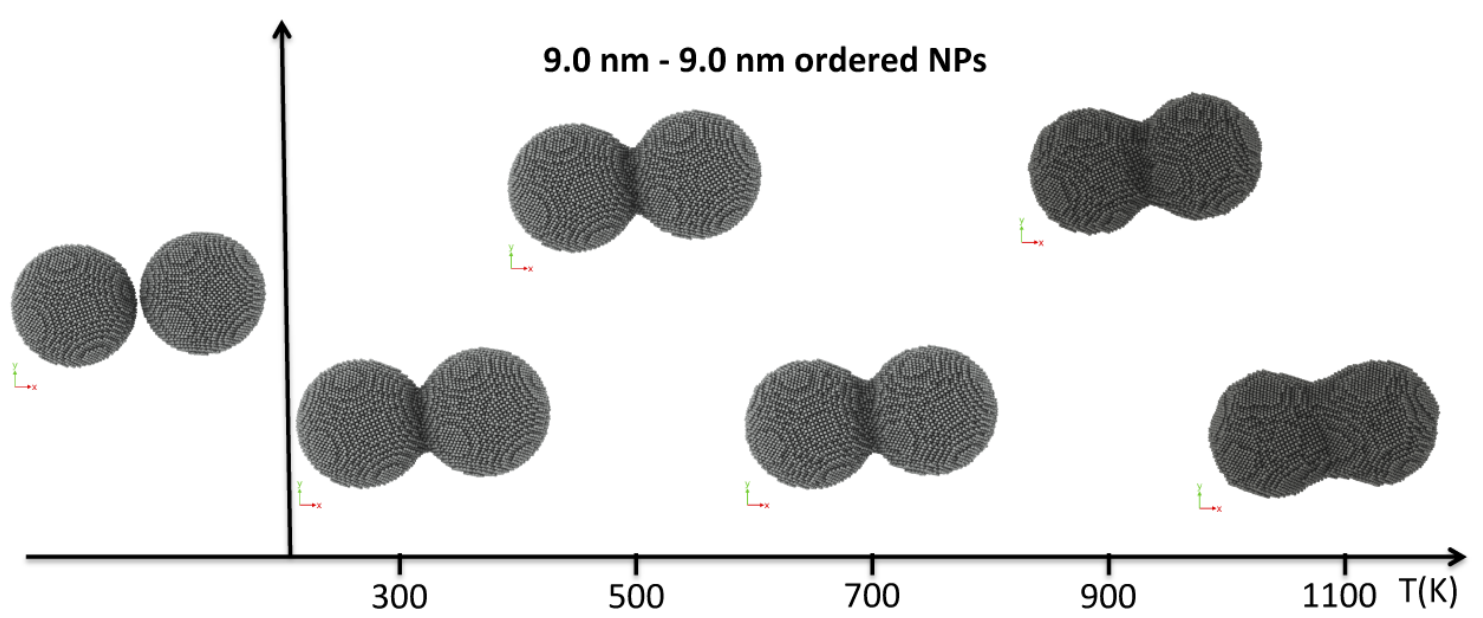

Figure 8: Temperature dependence of the coalescence process of two ordered Ag NPs of 9.0 nm.

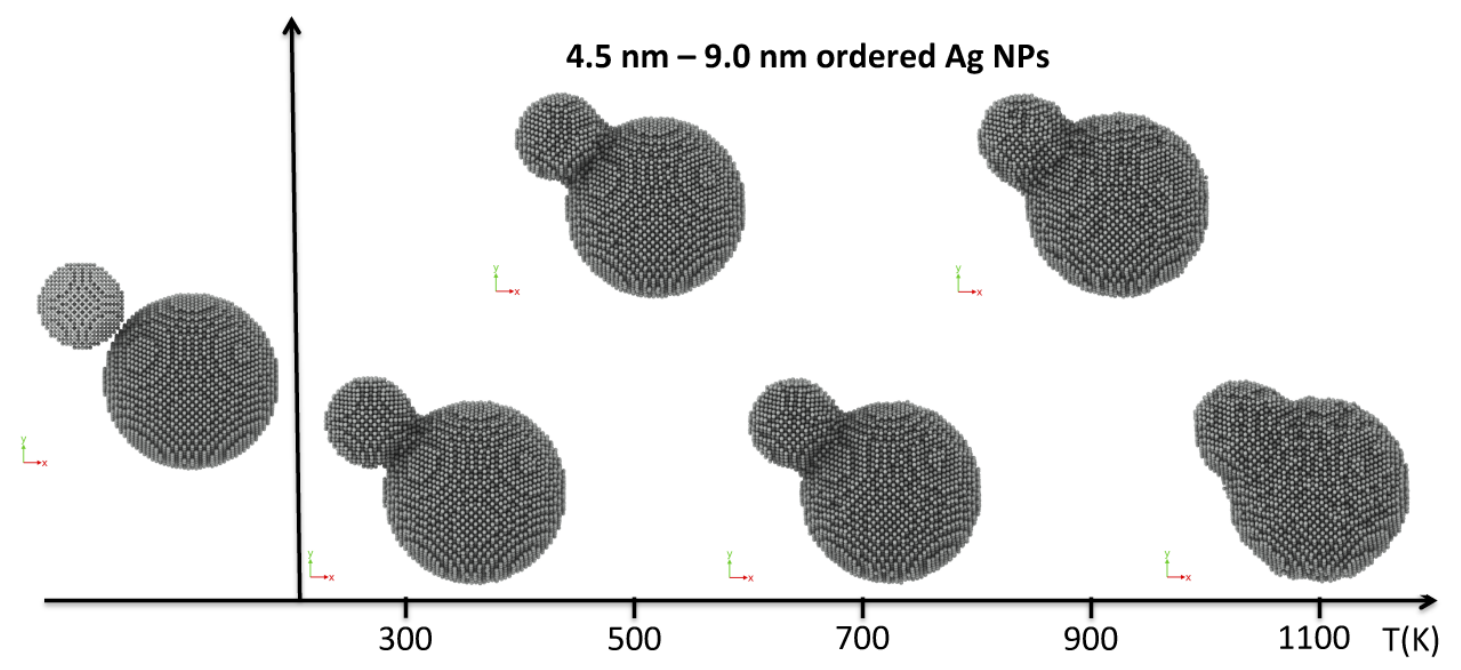

Figure 9: Temperature dependence of the coalescence process of two ordered Ag NPs of 4.5 $\mathrm{nm}$ and $9.0 \mathrm{~nm}$. 
coalescence shows an evolution that follows similar behavior as in the case of ordered NPs for the case of equal size $4.5 \mathrm{~nm}$ NP coalescence as can be observed in Fig. 10 . Figure 11

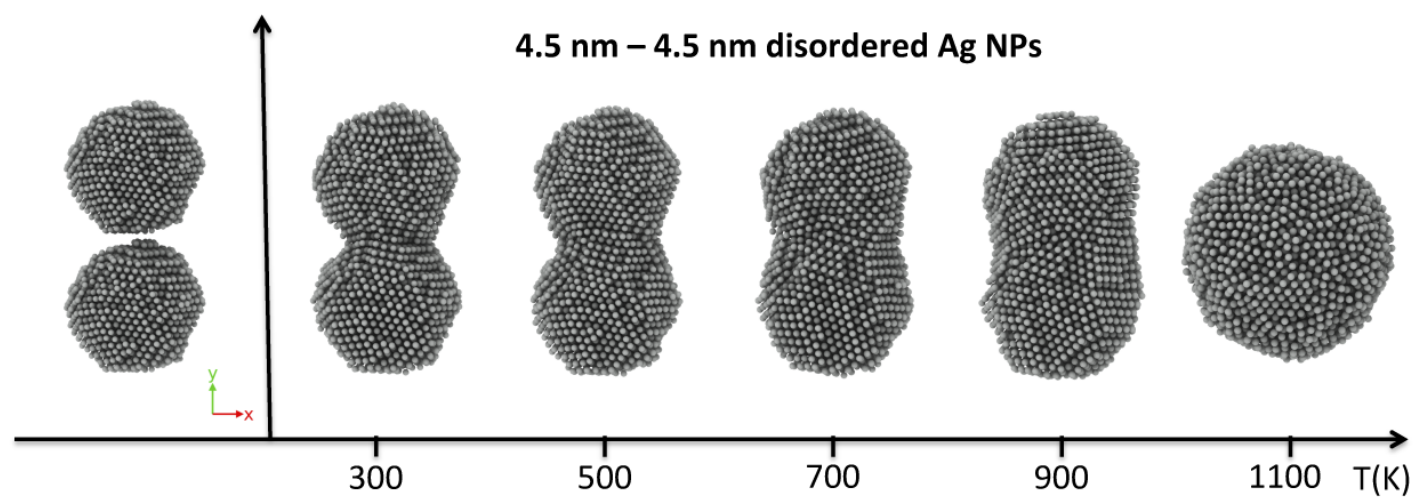

Figure 10: Temperature dependence of the coalescence process of two partially ordered Ag NPs of $4.5 \mathrm{~nm}$.

presents the temperature evolution of the 4.5-9.0 $\mathrm{nm} \mathrm{Ag} \mathrm{NPs} \mathrm{coalescence.} \mathrm{In} \mathrm{this} \mathrm{case} \mathrm{the}$

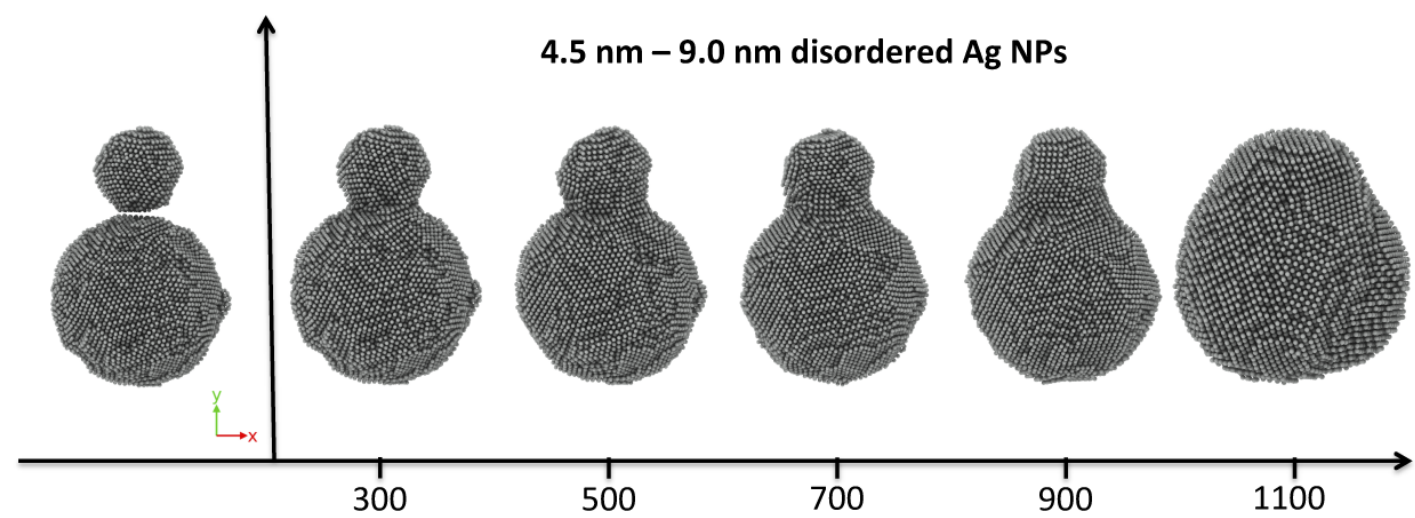

Figure 11: Temperature dependence of the coalescence process of two partially ordered Ag NPs of $4.5 \mathrm{~nm}$ and $9.0 \mathrm{~nm}$.

coalescence evolves more efficiently than in the case of ordered Ag NPs. The disordered Ag NPs evolve more easily into coalescence since being partially disordered from the start makes the effect of temperature more efficient when compared with the ordered case discussed in Fig. 9 . 


\section{Conclusions}

Motivated by the surprising experiments of Longo et al. ${ }^{5}$ where Ag NPs are produced in vacuum as result of electron beam irradiation from TEM on $\alpha-\mathrm{Ag}_{2} \mathrm{WO}_{4}$ crystals, our intention with the present work has been to study in greater detail the coalescence processes for Ag NPs using computer simulations. This represents an alternative to understand and describe this behavior as a clear example of SPR, based on well defined physical entities accessible from experiments. The same electron beam that produced these Ag NPs in the first place made them nano-electrical dipoles due to the electron magnetic field interaction forming SPR resonances in these NPs. Pairs of these neutral dipoles undergo interaction similarly to dimers and leading to coalescence when the interactions are attractive or moving away when interacting repulsively. The attractive dipole-dipole interactions initiate the coalescence processes experimentally observed by Longo et al. ${ }^{16}$ Computer simulations of molecular dynamics were used to investigate these processes. For that goal we have considered a variety of different configurations of interacting Ag NPs using equal and different sizes of Ag NPs. For the present study different degrees of order were considered, from crystalline to defective Ag NPs. In addition, the effect of temperature in the coalescence process was also investigated. Ordered Ag NPs tend to impose order, thus healing the disordered interface and stacking faults (hcp planes) as well as fcc order is forced upon the new structure. In the case of partially disordered Ag NPs, the recrystallization of disordered regions evolves better for larger NPs. The size effect prevents these mechanisms in the small $4.5 \mathrm{~nm} \mathrm{Ag}$ NPs. As expected, temperature helps to develop the coalescence process and disordered Ag NPs evolve faster to form the new merged Ag NPs.

\section{Acknowledgement}

The simulations were performed at the National Center for High Performance Computing in São Paulo (CENAPAD) and at the CCJDR-UNICAMP. We acknowledge support from 
FAPESP (2013/07296-2, 2015/19709-5). Partial support was provided by Capes and CNPq. J.A. acknowledges Generalitat Valenciana for Prometeo II/2014/022, ACOMP/2014/270, and ACOMP/2015/1202, and Ministerio de Economia y Competitividad (Spain) project CTQ2015-65207-P, Programa de Cooperación Cientifíca con Iberoamerica (Brasil) of Ministerio de Educación (Spanish Brazilian program PHBP14-00020), and Ministerio de Economia y Competitividad, "Salvador Madariaga" program, PRX15/00261,for financially supporting this research.

\section{Supporting Information Available}

The following files are available free of charge:

- SM-1: Sequence of snapshots of experiment of electron beam irradiation of two Ag NPs undergoing a coalescence process.

- SM-2: $g(r)$ for the coalescence process of two ordered Ag NPs with $9.0 \mathrm{~nm}$ of size.

- SM-3: $g(r)$ for the coalescence process of two ordered Ag NPs with sizes of $4.5 \mathrm{~nm}$ and $9.0 \mathrm{~nm}$.

- SM-4: $g(r)$ for the coalescence process of two disordered Ag NPs with $9.0 \mathrm{~nm}$ of size.

- SM-5: $g(r)$ for the coalescence process of two disordered Ag NPs with sizes of $4.5 \mathrm{~nm}$ and $9.0 \mathrm{~nm}$.

This material is available free of charge via the Internet at http://pubs.acs.org/.

\section{References}

(1) Jesse, S.; Borisevich, A. Y.; Fowlkes, J. D.; Lupini, A. R.; Rack, P. D.; Unocic, R. R.; Sumpter, B. G.; Kalinin, S. V.; Belianinov, A.; Ovchinnikova, O. S. Directing Matter: 
Toward Atomic-Scale 3D Nanofabrication. ACS Nano 2016, 10, 5600-5618, PMID: 27183171.

(2) Gonzalez-Martinez, I. G.; Bachmatiuk, A.; Bezugly, V.; Kunstmann, J.; Gemming, T.; Liu, Z.; Cuniberti, G.; Rummeli, M. H. Electron-beam induced synthesis of nanostructures: a review. Nanoscale 2016, 8, 11340-11362.

(3) Jiang, N. Electron beam damage in oxides: a review. Reports on Progress in Physics 2016, 79, 016501.

(4) Mansourian, A.; Paknejad, S. A.; Zayats, A. V.; Mannan, S. H. Stereoscopic NanoscalePrecision Growth of Free-Standing Silver Nanorods by Electron Beam Irradiation. The Journal of Physical Chemistry C 2016, 120, 20310-20314.

(5) Longo, E.; Cavalcante, L.; Volanti, D.; et al., Direct in situ observation of the electrondriven synthesis of $\mathrm{Ag}$ filaments on $\alpha-\mathrm{A} g_{2} \mathrm{WO}_{4}$ crystals. Scientific Reports 2013, 3, 1676.

(6) Andrés, J.; Gracia, L.; P., G.-N.; Longo, V.; Avansi, W.; Volanti, D.; Ferrer, M.; Lemos, P.; La Porta, F.; Hernandes, A. et al. Structural and electronic analysis of the atomic scale nucleation of $\mathrm{Ag}$ on $\alpha-\mathrm{Ag}_{2} \mathrm{WO}_{4}$ induced by electron irradiation. Scientific Reports 2014, 4, 5391.

(7) Pereira, W. d. S.; Andres, J.; Gracia, L.; San-Miguel, M. A.; da Silva, E. Z.; Longo, E.; Longo, V. M. Elucidating the real-time Ag nanoparticle growth on $\alpha$ - $\mathrm{A} g_{2} \mathrm{WO}_{4}$ during electron beam irradiation: experimental evidence and theoretical insights. Phys. Chem. Chem. Phys. 2015, 17, 5352-5359.

(8) San-Miguel, M. A.; da Silva, E. Z.; Zanetti, S. M.; Cilense, M.; Fabbro, M. T.; Gracia, L.; Andrés, J.; Longo, E. In situ growth of $\mathrm{Ag}$ nanoparticles on $\alpha$-A $g_{2} \mathrm{WO}_{4}$ under electron irradiation: probing the physical principles. Nanotechnology 2016, 27, 225703. 
(9) L., Y.; H., J. Y.; W., C.; S., S.; L., J. Cold welding of ultrathin gold nanowires. Nature Nanotechnology 2010, 5, 218-224.

(10) Zheng, H.; Cao, A.; Weinberger, C. R.; Huang, J. Y.; Du, K.; Wang, J.; Ma, Y.; Xia, Y.; Mao, S. X. Discrete plasticity in sub-10-nm-sized gold crystals. Nature Communications 2010, 1, 144 .

(11) Garnett, E. C.; Cai, W.; Cha, J. J.; Mahmood, F.; Connor, S. T.; Greyson Christoforo, M.; Cui, Y.; McGehee, M. D.; Brongersma, M. L. Self-limited plasmonic welding of silver nanowire junctions. Nature Materials 2012, 11, 241-249.

(12) Pereira, Z. S.; da Silva, E. Z. Cold Welding of Gold and Silver Nanowires: A Molecular Dynamics Study. The Journal of Physical Chemistry C 2011, 115, 22870-22876.

(13) Grouchko, M.; Roitman, P.; Zhu, X.; Popov, I.; Kamyshny, A.; Su, H.; Magdassi, S. Merging of metal nanoparticles driven by selective wettability of silver nanostructures. Nature Communications 2014, 5, 2994.

(14) Aabdin, Z.; Lu, J.; Zhu, X.; Anand, U.; Loh, N. D.; Su, H.; Mirsaidov, U. Bonding Pathways of Gold Nanocrystals in Solution. Nano Letters 2014, 14, 6639-6643.

(15) Grammatikopoulos, P.; Cassidy, C.; Singh, V.; Sowwan, M. a. Coalescence-induced crystallisation wave in Pd nanoparticles. Scientific Reports 2014, 4, 5779.

(16) Longo, E.; Avansi, W.; Bettini, J.; Andrés, J.; Gracia, L. In situ Transmission Electron Microscopy observation of Ag nanocrystal evolution by surfactant free electron-driven synthesis. Scientific Reports 2016, 6, 21498.

(17) da Silva, E. Z.; Faccin, G. M.; San-Miguel, M. A.; Andres, J.; Longo, E. Coalescence Process of Ag Nanoparticles. In Situ Visualization of Surface Plasmon Resonances. To be published. 
(18) Freestone, I.; Meeks, N.; Sax, M.; Higgitt, C. The Lycurgus Cup - A Roman nanotechnology. Gold Bulletin 2007, 40, 270-277.

(19) Huang, X.; El-Sayed, M. A. Gold nanoparticles: Optical properties and implementations in cancer diagnosis and photothermal therapy. Journal of Advanced Research 2010, $1,13-28$.

(20) Prodan, E.; Nordlander, P. Structural Tunability of the Plasmon Resonances in Metallic Nanoshells. Nano Letters 2003, 3, 543-547.

(21) Prodan, E.; Nordlander, P. Plasmon hybridization in spherical nanoparticles. The Journal of Chemical Physics 2004, 120, 5444-5454.

(22) Nordlander, P.; Oubre, C.; Prodan, E.; Li, K.; Stockman, M. I. Plasmon Hybridization in Nanoparticle Dimers. Nano Letters 2004, 4, 899-903.

(23) Koh, A. L.; Bao, K.; Khan, I.; Smith, W. E.; Kothleitner, G.; Nordlander, P.; Maier, S. A.; McComb, D. W. Electron Energy-Loss Spectroscopy (EELS) of Surface Plasmons in Single Silver Nanoparticles and Dimers: Influence of Beam Damage and Mapping of Dark Modes. ACS Nano 2009, 3, 3015-3022.

(24) Plimpton, S. Fast Parallel Algorithms for Short-Range Molecular Dynamics. Journal of Computational Physics 1995, 117, 1-19.

(25) Brown, W. M.; Wang, P.; Plimpton, S. J.; Tharrington, A. N. Implementing molecular dynamics on hybrid high performance computers - short range forces. Computer Physics Communications 2011, 182, 898-911.

(26) Brown, W. M.; Kohlmeyer, A.; Plimpton, S. J.; Tharrington, A. N. Implementing molecular dynamics on hybrid high performance computers - Particle-particle particlemesh. Computer Physics Communications 2012, 183, 449-459. 
(27) Brown, W. M.; Yamada, M. Implementing molecular dynamics on hybrid high performance computers-Three-body potentials. Computer Physics Communications 2013, $184,2785-2793$.

(28) Daw, M. S.; Baskes, M. I. Embedded-atom method: Derivation and application to impurities, surfaces, and other defects in metals. Phys. Rev. B 1984, 29, 6443-6453.

(29) Foiles, S. M.; Baskes, M. I.; Daw, M. S. Embedded-atom-method functions for the fcc metals Cu, Ag, Au, Ni, Pd, Pt, and their alloys. Phys. Rev. B 1986, 33, 7983-7991.

(30) Johnson, R. A. Alloy models with the embedded-atom method. Phys. Rev. B 1989, 39, 12554-12559.

(31) Mishin, Y.; Farkas, D.; Mehl, M. J.; Papaconstantopoulos, D. A. Interatomic potentials for monoatomic metals from experimental data and ab initio calculations. Phys. Rev. $B$ 1999, 59, 3393-3407.

(32) Sheng, H. W.; Kramer, M. J.; Cadien, A.; Fujita, T.; Chen, M. W. Highly optimized embedded-atom-method potentials for fourteen fcc metals. Phys. Rev. B 2011, 83, 134118.

(33) Sun, J.; He, L.; Lo, Y.-C.; Xu, T.; Bi, H.; Sun, L.; Zhang, Z.; Mao, S. X.; Li, J. Liquidlike pseudoelasticity of sub-10-nm crystalline silver particles. Nature Materials 2014, 13, 1007-1012.

(34) Lee, S.; Ryu, S. Molecular Dynamics Study on the Distributed Plasticity of PentaTwinned Silver Nanowires. Frontiers in Materials 2015, 2, 56.

(35) Filleter, T.; Ryu, S.; Kang, K.; Yin, J.; Bernal, R. A.; Sohn, K.; Li, S.; Huang, J.; Cai, W.; Espinosa, H. D. Nucleation-Controlled Distributed Plasticity in Penta-twinned Silver Nanowires. Small 2012, 8, 2986-2993. 
(36) Nosé, S. A molecular dynamics method for simulations in the canonical ensemble. Molecular Physics 1984, 52, 255-268.

(37) Nosé, S. A unified formulation of the constant temperature molecular dynamics methods. The Journal of Chemical Physics 1984, 81, 511-519.

(38) Hoover, W. G. Canonical dynamics: Equilibrium phase-space distributions. Phys. Rev. A 1985, 31, 1695-1697.

(39) Humphrey, W.; Dalke, A.; Schulten, K. VMD: Visual molecular dynamics. Journal of Molecular Graphics 1996, 14, 33 - 38.

(40) Varshney, A.; Brooks, F. P.; Wright, W. V. Computing smooth molecular surfaces. IEEE Computer Graphics and Applications 1994, 14, 19-25.

(41) Stukowski, A. Visualization and analysis of atomistic simulation data with OVITO-the Open Visualization Tool. Modelling and Simulation in Materials Science and Engineering 2010, 18, 015012 .

(42) Honeycutt, J. D.; Andersen, H. C. Molecular dynamics study of melting and freezing of small Lennard-Jones clusters. The Journal of Physical Chemistry 1987, 91, 4950-4963. 


\section{Graphical TOC Entry}

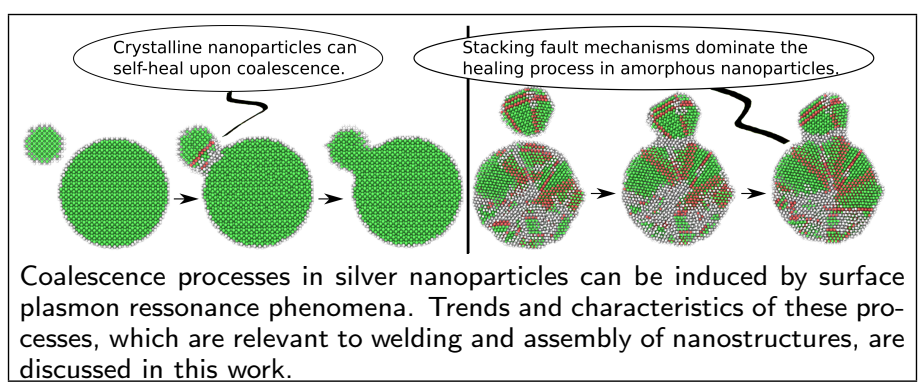




\section{Supplementary Material to:}

\section{A Computational Modeling for the Ag Nanoparticles}

\section{Coalescence Process: A Case of Surface Plasmon Resonance.}

SM-1
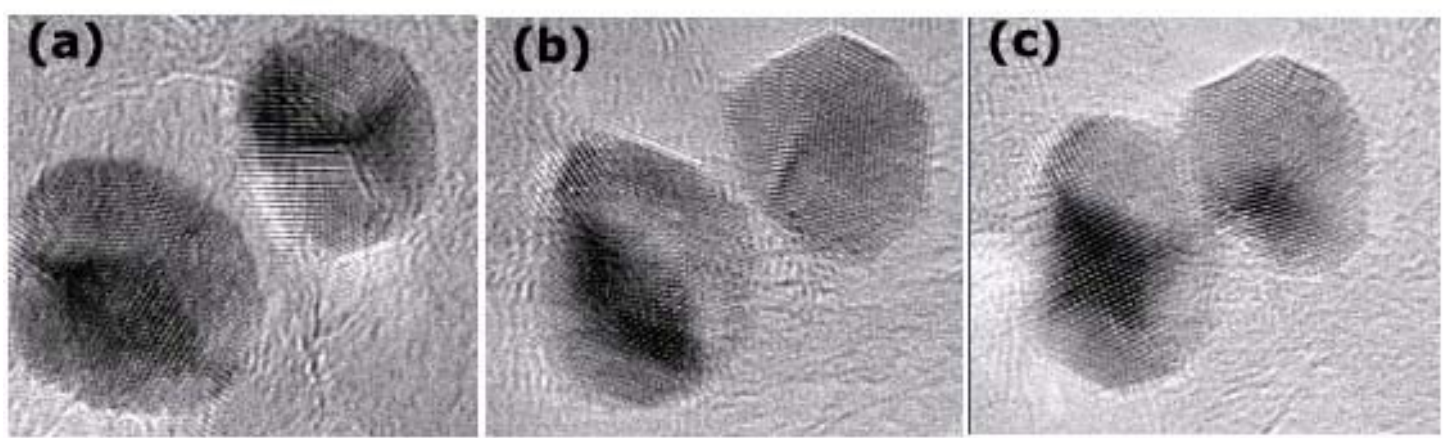

Figure SM-1. Sequence of snapshots of experiment of electron beam irradiation of $\alpha$ $\mathrm{Ag}_{2} \mathrm{WO}_{4}$. These two NPs, sizes $9 \mathrm{~nm}$ and $6 \mathrm{~nm}$ of radii, undergo a coalescence process similar to some simulations performed in the present article. 


\section{SM-2}

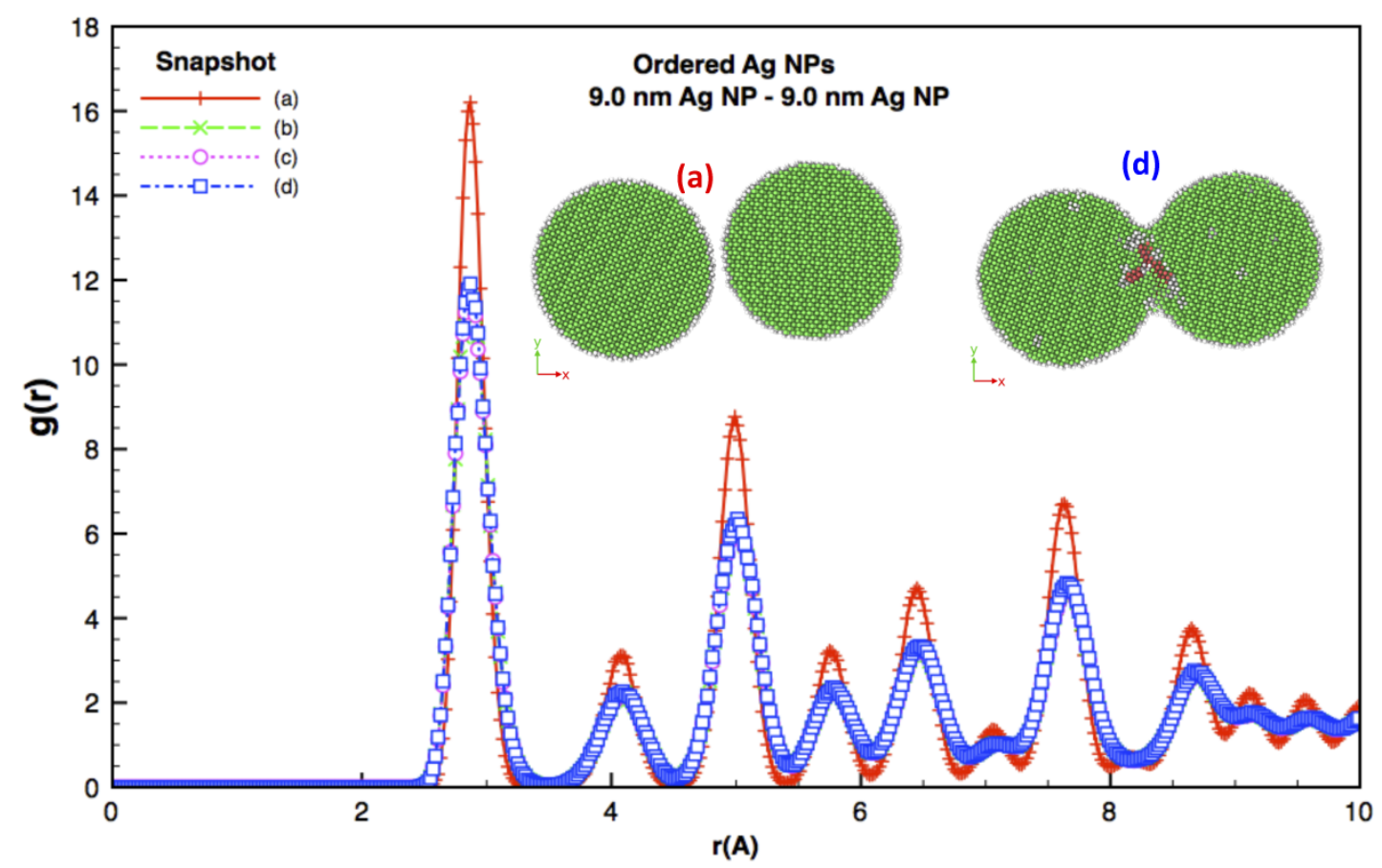

Figure SM-2 The radial distribution function $\mathrm{g}(\mathrm{r})$ of the simulation of the coalescence process of two ordered $9.0 \mathrm{~nm} \mathrm{Ag} \mathrm{NPs} \mathrm{at} 500 \mathrm{~K}$. The g(r) labels correspond to the snapshots displayed in Fig-2. Insets display the initial (a) and the final (d) simulation snapshots. 
SM-3

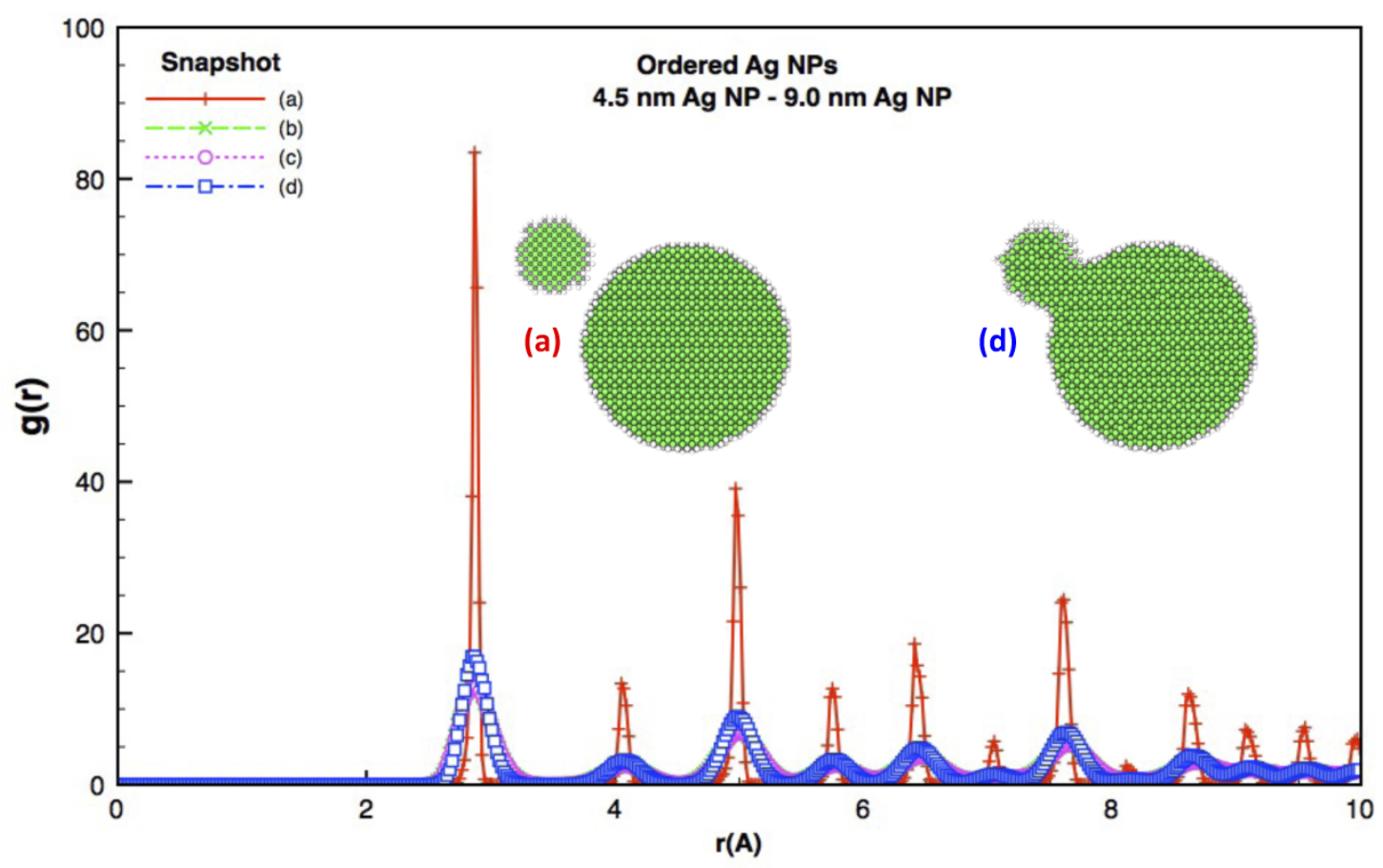

Figure SM-3 The radial distribution function $\mathrm{g}(\mathrm{r})$ of the simulation of the coalescence process of a 4.5 and $9.0 \mathrm{~nm}$ ordered Ag NPs at $500 \mathrm{~K}$. The g(r) labels correspond to the snapshots displayed in Fig-3. Insets display the initial (a) and the final (d) simulation snapshots. 


\section{SM-4}

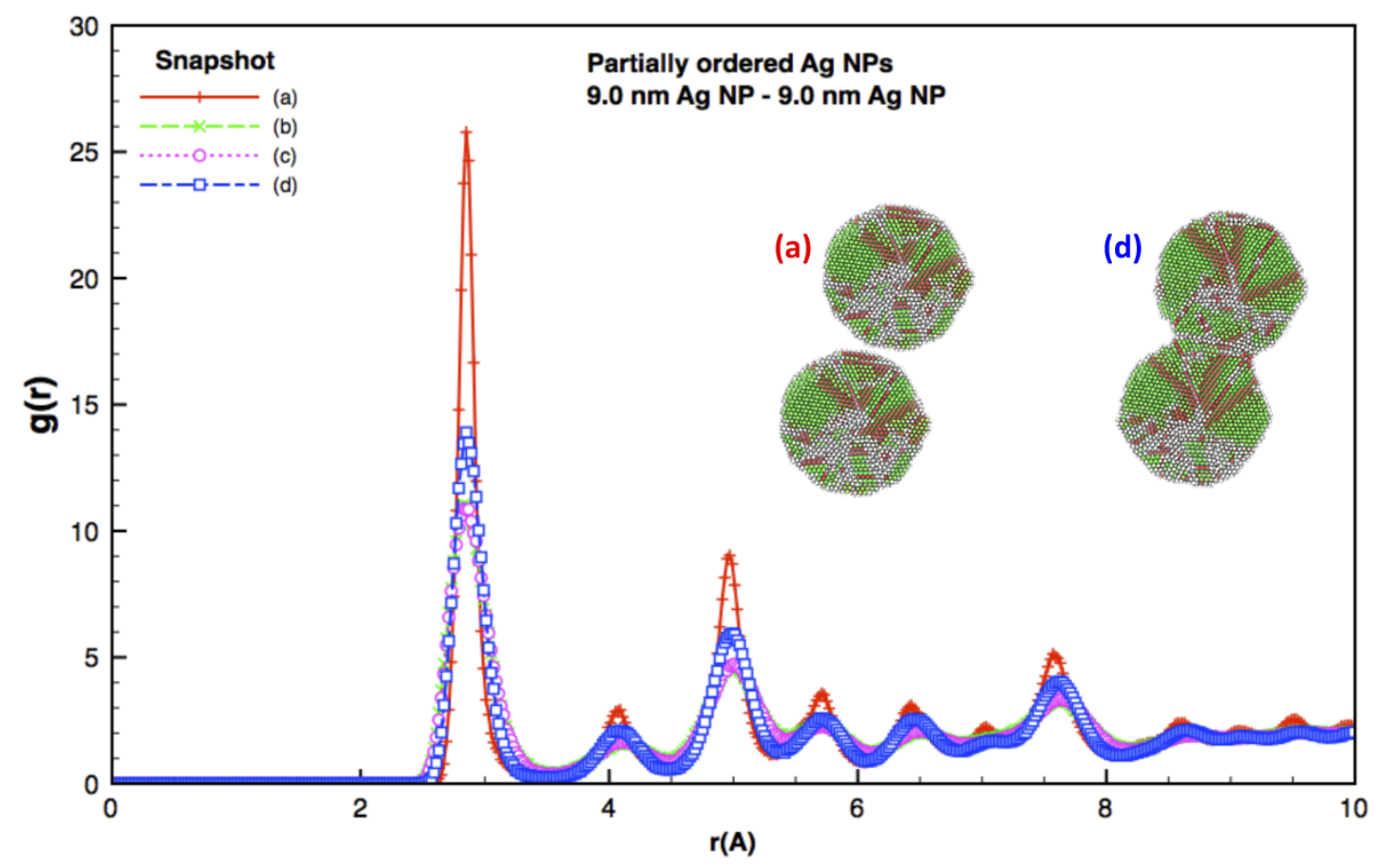

Figure SM-4 The radial distribution function $\mathrm{g}(\mathrm{r})$ of the simulation of the coalescence process of two $9.0 \mathrm{~nm}$ disordered Ag NPs at $500 \mathrm{~K}$. The g(r) labels correspond to the snapshots displayed in Fig-5. Insets display the initial (a) and the final (d) simulation snapshots. 


\section{SM-5}

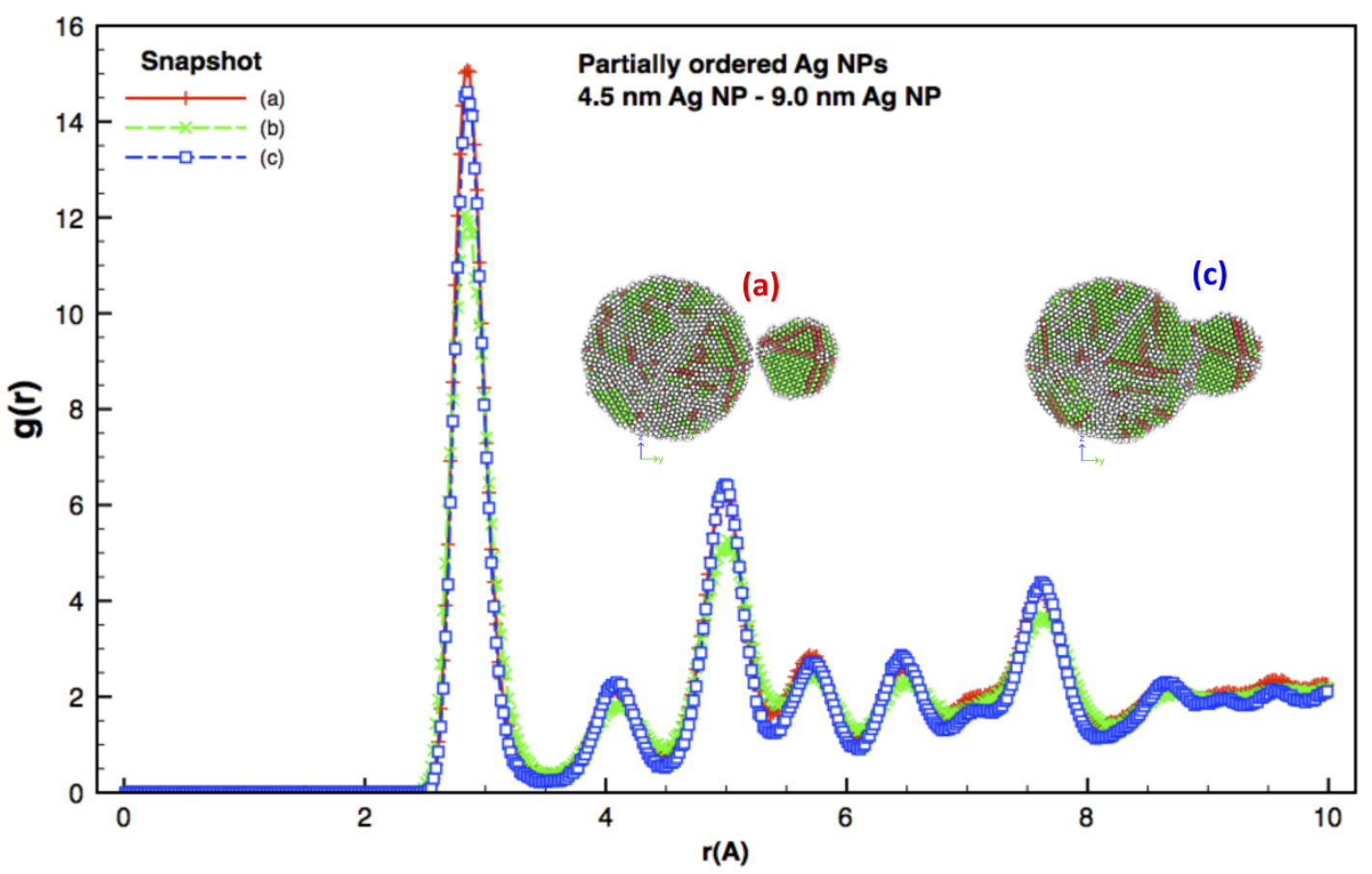

Figure SM-5 The radial distribution function $\mathrm{g}(\mathrm{r})$ of the simulation of the coalescence process of a 4.5 and a $9.0 \mathrm{~nm}$ disordered Ag NPs at $500 \mathrm{~K}$. The $\mathrm{g}(\mathrm{r})$ labels correspond to the snapshots displayed in Fig-6. Insets display the initial (a) and the final (c) simulation snapshots. 